Journal of Research in Interprofessional

Practice and

Education

Vol. 4.1

June 2014
Journal of Research in Interprofessional Practice and Education (JRIPE)

Vol. 4.1

(c) 2014

Corresponding author: Marianne Solberg. Email:m.t.solberqi @-@edisin.uio.no!

\section{Oxygen and Ventilator Treatment: Perspectives on Interprofessional Collaboration in a Neonatal Intensive Care Unit}

\author{
Marianne Trygg Solberg, RN, MNsc; Thor Willy Ruud Hansen, MD, \\ PhD, Professor; \& Ida Torunn Bjørk, RN, PhD, Professor
}

\begin{abstract}
Background: The aim of this study was to explore perspectives on the collaboration between physicians and nurses managing oxygen and ventilator treatment of sick infants in a Norwegian neonatal intensive care unit.

Methods and Findings: We performed a qualitative study using focus groups. We found that interprofessional collaboration concerning newborns on mechanical ventilation lacked co-ordination and was unsystematic. This led to inadequate utilization of the medical and clinical competency of the nursing staff. Nurses and physicians approached decision-making differently, and there was limited flexibility and dynamics in the allocation of responsibility between the professionals.

Conclusion: Findings from this study indicate that nurses and physicians have the opportunity to improve the quality of care by developing high-quality communication, formulating plans together, and improving the co-ordination of the ventilator treatment. Further studies should develop and test interventions based on the professionals' perception of relevant co-ordination strategies to improve mechanical ventilation and oxygen treatment to premature and sick newborn infants.
\end{abstract}

Keywords: Quality of care; Collaboration; Oxygen treatment; Mechanical ventilation; Neonatal intensive care unit

\section{Introduction}

The management of oxygen and ventilation therapy in mechanically ventilated premature and sick newborn infants is a complex task that requires collaboration between nurses and physicians [1]. Collaboration and quality of care are closely connected, and improved collaboration reduces errors, adverse events, and length of stay [2]. Conversely, inadequate interprofessional collaboration has been associated with medication errors, patient safety problems, and deaths [3]. Interprofessional collaboration is important for improving the effectiveness of healthcare services. Communication is essential in collaboration because it is necessary for all collaborators to understand each other to reach a goal [4]. Therefore, collaboration may be improved by improving communication. Numerous studies have concluded that more effective communication between nurses and physicians could improve the quality of care [5-7]. Patient care has been shown to be more effectively co-ordinated when a high quality of relationships and communication was present in the decision-making process [8].

Collaboration in the ventilation and oxygenation of premature and newborn infants has not been investigated, to our knowledge. Studies on oxygen and ventilator treatment have mainly focused on possible tissue damage and the development of sat- 
2

Collaboration on Ventilation of Newborns

Solberg, Hansen, \& Bjørk

Journal of Research in Interprofessional Practice and Education

Vol. 4.1

June 2014 uration limits to guide oxygen titration [9,10]. In general, collaborative decision-making processes are inadequate in neonatal intensive care units (NICUs) [11-13]. The literature emphasizes the nurses' view, but it is also important to report on physician experiences [3]. To provide a more complete picture of the decision-making process in NICUs, Dunn et al. [11] recommended qualitative studies using focus groups to explore professional perspectives. This study is the second part of a larger study in a Norwegian NICU that aims to discover areas for potential quality improvement regarding oxygen and ventilator treatment of preterm and sick newborn infants. The aim of the present study was to use focus group interviews to explore how physicians and nurses experienced their collaboration when working with oxygen and ventilator therapy.

\section{Challenges related to collaboration and responsibility}

In a NICU setting, collaboration to provide secure medical care is the primary concern [14], and in newborn infants who receive mechanical ventilation, the goal is to balance gas exchange while causing as little damage as possible [15]. Studies have found that collaboration was influenced by the clinical experience of nurses and physicians, different applications of knowledge, the use of cognitive intuition, and the difference in how professionals interpreted research findings [16]. Insufficient use of guidelines impeded collaboration and hindered maintenance of adequate oxygenation targets [17].

Several barriers to good interprofessional relationships in collaboration have been identified, including time constraints, a lack of understanding of other's roles and tasks, poor organizational support or intolerant power structures, different traditions, professional values, different goals, and different priorities [18]. Collaboration also depended on individual characteristics and perceptions about working together in a team [2]. Unequal group power distribution in the hospital hierarchy influenced communication between professionals in a team [2], and physicians historically receive more respect than nurses [3]. Nurses and physicians may disagree on the interpretation of "good work" and the implications of their responsibilities although they acted in a professionally responsible manner [19]. The professional responsibilities of consultants in the NICU include providing care and offering supervision for the neonatology fellows/registrars, house officers/residents, and nurses [14]. Nurses are responsible for oxygen administration, applying ordinations in practice [16], the implementation of care plans, and assisting in medical care $[14,3]$. Professional responsibilities in the NICU involve trust, which is fundamental to interprofessional relationships in collaboration [20,21]. Trust is an important element in collaboration and takes time to achieve between professionals [14]. Trust is achievable with sufficient competence, shared goals, professional boundaries, and established patterns of collaboration [21].

\section{Theory of cognitive collaboration}

Several definitions of the term "collaboration" have been presented to explain interprofessional collaboration between physicians and nurses. The core concept of col- 
3

Collaboration on Ventilation of Newborns

Solberg, Hansen, \& Bjørk

Journal of Research in Interprofessional Practice and Education

Vol. 4.1

June 2014 laboration is characterized in the literature with elements of sharing, partnership, power, interdependency, process, and dynamics within a given context between professionals $[22,4]$. Collaboration is synonymous with co-operation and is defined by Hoc [4] as "acting together" to reach a goal that is shared by the co-operating individuals. Hoc's [4] theory of co-operation can illuminate important aspects of physicians' and nurses' collaboration in acute and dynamic situations toward the goal of superior oxygen and ventilator treatment to premature and newborn infants. Hoc's [4] theory is used to inform our views on collaboration in this article. Although Hoc uses the term "co-operation," we consistently use the term "collaboration."

Collaborative activities between individuals occur in dynamic situations and should have a prominent cognitive aspect [4]. There are three levels of collaboration: 1) in planning, 2) in action, and 3) at the meta level. Collaboration at the planning level aims to share contextual statements, which improves communication and the choice of plans. At this level, co-ordination is an important component of collaborative activities and can be described as unilateral (one agent changes the plan), bilateral, or mutual with conscious co-ordination intent and agreement [4]. At the action level, the collaborating individuals regularly update their knowledge and perception as the collaborative activities are related to goals and how to manage procedures [4]. Collaboration at the meta level signifies that clinicians can produce useful tools and guidelines for their collaborative activities. Hoc [4] states that communication is necessary to understand others, and using codes as an "operative language" in collaboration is helpful because they are formal and involve restrictions to the clinicians' natural language. Consequently, using an operative language with communication codes allows the extent of communication to be reduced, and professionals save time while communicating [4].

\section{Theory of relational co-ordination}

We use Gittel's [23] measure of relational co-ordination to describe and assess coordination between physicians and nurses because communication and quality of care are improved as a result of relational co-ordination [24-26,2]. Before Gittel studied the effect of relational co-ordination in healthcare settings, she validated the theory in airline flight departure processes. Relational co-ordination is the management of tasks between professionals, and the theory describes the relationships between roles rather than individuals. Relational co-ordination emphasizes that high quality communication should be characterized by frequent, timely, and accurate communication focused on problem solving, while the co-ordinated action should be based on shared goals, shared knowledge, and mutual respect to strengthen the relationships [23]. Gittel [23] defines 12 high-performance work systems that help strengthen the relations and systematically co-ordinate work between physicians and nurses. In this article, we apply four of Gittel's work systems when discussing the results: broadening participation in patient rounds, selecting teamwork skills, rewarding team performance, and improving the flexibility of job boundaries [23]. 
4

Collaboration on Ventilation of Newborns

Solberg, Hansen, \& Bjørk

Journal of Research in Interprofessional Practice and Education

Vol. 4.1

June 2014

\section{Methods}

Design

We developed an exploratory qualitative design including four focus group interviews during the period of September through October 2012 at Oslo University Hospital, Rikshospitalet. An exploratory design is frequently used to understand the perspectives of professionals on a problem and its cause [27]. The exploratory design in this study involves an analysis of current practice and was chosen because the evidence from the literature is insufficient [27].

\section{Participants and setting}

NICUs in Norway employ only physicians and nurses as caregivers. Nurses are registered nurses, and may or may not have a pediatric or intensive care specialty. There are no respiratory therapists or nutritional experts employed in NICUs. The NICU used in this study is a large unit with 120 nurses (non-specialist nurses and specialist nurses in intensive, pediatric, or neonatal care) and ten physicians (seven consultants, one registrar/fellow, and two house officers). The study included 20 participants with varied background and experience to increase the likelihood of contrasting opinions [28]. We used purposeful sampling based on principles from maximum variation sampling [29]. The logic of maximum variation sampling is to use different dimensions of each participant to strengthen the results because common patterns emerge from a variety of participant experiences [29]. The different dimensions of participants in this study were stage in career, age, and expertise in both professions. Nurse participants were recruited by a charge nurse on three specified days. All nurses on day and afternoon shifts were asked to participate, and volunteers were included. Physicians were recruited by a consultant working in the NICU. All employed physicians were emailed about the study. Three focus groups consisted of four, seven, and five nurses (all female), and one group consisted of four physicians (one female and three male). The participants' experience in the NICU varied from 1.5 to 34 years among the nurses, and although the physicians reported experience from a few months to seven years in the NICU, they reported work experience as physicians from 7 to 22 years.

\section{Data generation}

We conducted focus group interviews to gather information from participants. A focus group interview can provide the participants opportunities to discuss opinions and experiences of a given topic without necessarily formulating a consensus $[28,30]$. Group dynamics might stimulate spontaneous expressive and emotional views [30] and provide more information than individual interviews [28]. We invited a small number of people in each group, because when the topic is complex and the participants' level of expertise is high, the literature recommends limiting the number of people in the group [28]. In this study we chose to interview physicians and nurses separately. We were interested in similarities and differences in experience (the gap) between professions and did not want to risk undue influence 
5

Collaboration on Ventilation of Newborns

Solberg, Hansen, \& Bjørk
Journal of Research in Interprofessional Practice and Education

Vol. 4.1

June 2014 by one or the other of the professions in the discussions. To be consistent during the data collection, we used a semi-structured question guide with open-ended questions based on the review of the literature and the results of Study 1, regarding adherence to oxygenation and ventilation targets [1]. The topic guide was validated in discussions with a physician and with nurse instructors employed in the study NICU as well as the other NICU currently operating at Oslo University Hospital, (location Ullevall). The topic guide was similar for nurses and physicians and included an open introduction asking for descriptions of challenging situations when they managed oxygenation and ventilation. The core questions addressed responsibility and communication, for example, how do you think responsibilities in ventilator treatment in the unit are functioning? When the participants discussed issues related to the results in Study 1 [1], the findings were introduced by the moderator into the discussion. The first author moderated the focus group interviews, and the senior author observed and noted topics that might be expanded on at the end of the interview. The focus group sessions lasted 60 minutes and were audiotaped and transcribed verbatim.

\section{Ethics}

Permission for the study was obtained from the data protection officer at Oslo University Hospital and from the director of the NICU. In accordance with the Declaration of Helsinki, participation was voluntary. Respondents could withdraw from the study at any time, no identifying characteristics were audiotaped to maintain anonymity, and the participants were assured full confidentiality. Approval by the regional committee for medical research ethics in Norway was not required for this study.

\section{Trustworthiness}

Opinions stated in the results were constructed in a social situation, with discussions occurring in a specific, controlled setting [31]. Moderator bias was minimized because the moderator (first author) was from a background similar to the participants. There were not many dominant voices overriding other voices, but since the participants had different experiences and roles in the ward, the moderator actively encouraged all group members to speak. We experienced a good atmosphere with much humour during the discussions. The participants were engaged and not afraid to express different opinions about problems, frustrations, as well as good collaboration they experienced. The first author strived to explicate both coinciding and divergent views from the participants.

\section{The analysis}

We used content analysis to explore the meanings in the communications of nurses and physicians. The analysis was inspired by Kvale and Brinkmann [30], and was performed using five steps to systematically find similarities and differences between the professionals. In the first step, we observed each focus group interview followed by a debriefing between the moderator and the observer. The audiotapes were tran- 
Collaboration on Ventilation of Newborns

Solberg, Hansen, \& Bjørk
Journal of Research in Interprofessional Practice and Education

Vol. 4.1

June 2014
Journal of Research in Interprofessional Practice and Education

scribed, and the transcripts were read several times to gain insight into the content before the text was condensed into meaning units. The second step consisted of a data-driven coding to classify the meaning units. In data-driven coding, no codes are defined beforehand, and the codes are inductively developed by reading and rereading the meaning units [30]. The codes were used as tools to investigate the manifest content to identify more overall themes with a higher level of abstraction [30]. In the third step, all the meaning units and their codes were placed into subcategories. In the fourth step, a descriptive summary was written of each subcategory developed in the three interviews with the nurses and the interview with the physicians to compare and look for contrasts between the professionals [28]. In the fifth step, posters were made that displayed all the subcategories to develop more abstract themes. The posters visualized the subcategories and their units of meaning and were helpful in identifying structures and the relationship between meanings beyond the direct transcript of the interviews [30]. Using this process, the results were interpreted by looking for patterns related to collaboration, communication, and responsibility. Table 1 shows the themes with their subcategories.

\section{Table 1}

\section{Themes with subcategories}

Perspectives on collaboration and communication

- The collaboration characterized

- The communication characterized

- Communication with registration of patterns, who took preliminary contact

Co-ordination of the ventilator treatment

- How caregivers' competence affects decisions

- Physician-selected mode

Utilization of nurses' competence

- Teamwork

- Information exchange

- Factors promoting clinical decisions

- Factors preventing clinical decisions

- Technical equipment

- Mutual respect, trust

Perceptions of inter- and intraprofessional responsibility

- Perceived responsibility

- Flexible responsibilities

The professional approaches in decision-making

- How clinical decisions are made

- Factors influencing clinical decisions

- Medical and clinical knowledge

- Knowing the child

- Relationships between professions 
7

Collaboration on Ventilation of Newborns

Solberg, Hansen, \& Bjørk

Journal of Research in Interprofessional Practice and Education

Vol. 4.1

June 2014

\section{Results}

The findings are presented according to the five themes that emerged from the analysis. To ensure anonymity, each participant's citation is coded with a non-identifying number representing the focus group and the individual (e.g., 3-2).

\section{Perspectives on collaboration and communication}

Both physicians and nurses perceived collaboration to be varied and considered communication to be the most important element when collaborating. They divulged that communication was not ideal between some of the nurses and some of the physicians due to personality conflicts. The physicians had more positive perceptions of the communication than nurses, and one of the physicians related the characteristic of their communication to the nurses' expertise in the following way:

What I have learned is that once you become familiar, you know who has the expertise and you trust them when caring for a sick baby. It is another situation all together when an inexperienced nurse is responsible for a ventilated baby or when a nurse in training is bedside. Then the communication is a bit different, and that is the way it has to be. (3-3)

The nurses commented that the collaboration between nurses also varied and identified that good collaboration involved aspects of mutual communication and afforded sympathy, generosity, and mutual respect. One experienced nurse said "I think we have it quite fine here, but the communication is something we want to work on; it is not good" (4-3). Some physicians strongly voiced their opinions and gave nurses no leeway for discussions. Several nurses felt uncomfortable questioning the physicians about the treatment, and other nurses reported contacting the physicians and not receiving answers to their questions.

Both physicians and nurses agreed that there was a great workload in the unit. Instances of failed collaboration occurred daily due to a lack of discussion between the professionals on how to determine the optimal ventilator setting for newborns. In contrast to the nurses' experiences, one of the physicians mentioned that the youngest and least skilled nurses should ask questions. The physician indicated that inexperienced nurses mostly asked about saturation targets, while the experienced nurses asked about more challenging situations. The physicians' perceptions differed regarding the nurses' courage to communicate with the physicians: "It may be difficult to ask you as a 'great doctor' relative to me as a 'little doctor"' (3-4). The nurses preferred to collaborate with the consultants, who were at the top of the hierarchical structure, whereas the house officers preferred to ask nurses, who were lower in the hierarchy. The house officers asked the consultant only if they were unsure about the treatment. In summary, communication concerning the ventilator treatment suffered because nurses and physicians did not communicate within a team but in a unidirectional manner with little reciprocity. Nurses took much of the responsibility for the fluidity of the communication between the professionals and initiated the contact with physicians based on their consideration of the need to adjust the ventilator setting. 
8

Collaboration on Ventilation of Newborns

Solberg, Hansen, \& Bjørk

Journal of Research in Interprofessional Practice and Education

Vol. 4.1

June 2014

\section{Co-ordination of the ventilator treatment}

A central finding was that both nurses and physicians were frustrated by the exercise of discretion in the ventilator treatment, which can be characterized as a physician-selected treatment with a setting that differed from shift to shift. The underlying norm for the ventilator treatment was that physicians did their best to decide the setting without guidelines and attempted to understand what to do and when to do it. One of the physicians explained:

Perhaps you can reach the goal in different ways, but I have experienced that on Saturday we run the treatment with one setting, then on Sunday there is another consultant on duty, and then we run a completely different setting. (3-4)

The nurses addressed the same issue:

Sometimes we experience that the physician on duty decides on a certain mode. The next day, and this is especially true during holidays, another physician comes along and switches to SIPPV, so that in a week the poor infant has ... I don't know how many settings we have on the ventilator. It's like "now I am on duty, and this is the way I want to have it," and I think that's very frustrating. Actually, just the fact that the physician "thinks it's right" is not a good enough argument. (4-3)

One physician proposed that they should use algorithms, while another argued the need to use experience and feelings in decision-making. The nurses expressed a clear opinion that both nurses and physicians should collaborate in planning the ventilator setting. They wanted to be heard, but in some situations, the nurses felt powerless (e.g., when physicians changed the ventilator setting as suggested by the nurse, but only after the nurse left the shift). The results of the interview illustrate that there were no common goals or guidelines to co-ordinate the ventilator treatment to ensure continuity for the sick newborns.

\section{Utilization of nurses' competence}

The physicians and nurses reported some discussions, but the nurses felt that they had few opportunities to discuss and formulate plans with physicians. In this NICU, a pre-round conference between physicians and the leading nurses was performed before the physician rounds for the sick newborn infants. Most of the nurses had never attended this pre-round conference and considered this to be problematic because they were not aware of the physician's considerations. The nurses wanted to be included in the planning process with physicians to understand the reasons behind the treatment. In addition, nurses wanted predetermined limits to guide oxygenation and ventilation management because the opinions of the physicians often differed:

There was one physician who said that the baby should have above 95\% (saturation), then came the second physician and said "Why 
9

Collaboration on Ventilation of Newborns

Solberg, Hansen, \& Bjørk

Journal of Research in Interprofessional Practice and Education

Vol. 4.1

June 2014 should this baby have oxygen, he does not need to be so high, not more than 90\%." This can sometimes be a bit difficult to deal with. (2-7)

In the physicians' opinions, the nurses had more education regarding ventilator treatment and lung function in premature and newborn infants than the house officers. The house officers often made changes in the ventilator settings based on suggestions from the nurses. One physician remarked on the importance of trusting the nurses to give newborns the best treatment:

They tell us about oxygen saturation, they tell us about the tidal volume and triggering. They know what they're doing and can provide a great deal of feedback. Nurses state that "this is what the situation is like now," not only that the infant has kept a low or high saturation, but also good information about the lung situation. (3-1)

This statement showed how an experienced physician judged the nurses to be competent regarding ventilator treatment and that nurses were in possession of medical information that was important for collaboration in the decision-making process.

The nurses in all the focus groups talked repeatedly about the importance of knowing the infant. They developed an accurate knowledge of the newborns' response to the treatment, which they had few opportunities to articulate. Although the physicians in the focus group recommended listening to the nurses, nurses stated that it was challenging to gain trust in certain cases or responsiveness to their opinions. The nurses argued that physicians had to rely on their observations because they included more than the numbers on an acid-base slip (e.g., when an infant is uncomfortable with the ventilator setting). The nurses felt that they were heard if they had relevant medical knowledge to contribute. The nurses expressed a strong attachment to the medical knowledge without having any leeway in using that knowledge.

\section{Perceptions of inter- and intraprofessional responsibility}

Nurses' and physicians' views on inter- and intraprofessional responsibility regarding ventilator and oxygen treatment coincided. Physicians decided on the ventilator setting and limits for oxygen and specified and executed changes. They felt responsible for the low $\mathrm{PaCO}_{2}$ values presented in Study 1 [1], and reflected on the lack of routines in prescribing tidal volumes. One resident wanted a discussion on whose responsibility (resident or consultant) it was to change the ventilator setting on the basis of very small margins in the treatment of the extremely premature.

Nurse responsibilities were oxygen regulation and consideration of the need for change in the ventilator setting. They maintained free airways, kept endotracheal tubes securely attached, and managed fluctuations in saturation. Nurses suggested that their responsibility in caring for premature and sick newborn infants should be differentiated according to observation skills and experience. One experienced nurse declared that even with thorough ventilator training, it was sometimes difficult to know when a new nurse could be entrusted with more responsibility. Nurses 
10

Collaboration on Ventilation of Newborns

Solberg, Hansen, \& Bjørk
Journal of Research in Interprofessional Practice and Education

Vol. 4.1

June 2014 said that their care for the sick premature and newborn infant was at the "frontline." They characterized this frontline work as assessing acid/base tests, tidal and minute volume, and setting alarm limits for pulse oximetry and the ventilator. All observations of technical, perceptual, and interactive cues had to be documented. This frontline work was acknowledged by the physicians giving examples such as nurses requesting an acid/base test or requesting to change low ventilator rates, when an infant's saturation decreased during sleep.

Flexible responsibility was discussed in all groups. The nurses and physicians agreed that nurses were given more responsibility in other intensive care units, and more responsibility was given to the NICU nurses if the physicians were familiar with the nurse and the nurse's level of competence. One of the physicians reported that setting the ventilator treatment was a great responsibility because the lung function of an extremely preterm infant is a decisive factor for survival. An increase in the nurses' responsibilities was therefore considered problematic. Because of these challenges, the perspectives on flexible responsibility in reducing or increasing the peak pressure differed. One physician had never experienced nurses regulating the peak pressure, while another physician said "There are a handful of nurses who have sufficient knowledge of tidal volume and minute volume. They make suggestions, and then they also reduce" (3-3). Some nurses stated that if they had to wait for adjustments, the physician gave them more responsibility and told them to turn down the peak pressure.

Because the physicians had a high workload most of the time, the nurses had to wait for changes in the ventilator setting:

There can be a lot of waiting, which can affect the infant, because the physicians have so much to do. You become impatient, like when they are attending to a recently admitted newborn infant. You do not want to interfere, and you know about the difficulties they have to manage, so I would like to have a little more flexibility in my responsibilities. (1-4)

The perceptions of what constituted a "long time" to wait for corrections of the ventilator setting depended on the infants' level of acuity. Nurses mentioned waiting times from 30 minutes to 1.5 hours. For extubation, the nurses reported waiting from the night shift to the day shift. To reduce the ventilator time and length of stay, many of the nurses wanted more flexibility to perform necessary adjustments exercised on the basis of guidelines. Some nurses would like to perform extubation when prescribed, and one nurse concluded, "We can only become more competent if we are given responsibility" (1-4).

\section{Professional approaches in decision-making}

The data provided insight into how nurses used both medical and clinical knowledge when managing ventilator treatment. Regarding medical knowledge, the nurses explained that rapid changes in oxygen could cause hyperoxia, and rapid changes in $\mathrm{PaO}_{2}$ could cause damage. Clinical knowledge, such as knowing the newborn infant, 
11

Collaboration on Ventilation of Newborns

Solberg, Hansen, \& Bjørk
Journal of Research in Interprofessional Practice and Education

Vol. 4.1

June 2014 took a day to establish, and this knowledge was most important in the nurses' decision-making process. Because nurses typically managed only one critically ill newborn infant, the nurse obtained a long-term view of the infant, while the physician had little opportunity to observe the patient over time. When nurses knew the infant, the oxygen therapy could be individualized because the nurses knew how low the saturation could fall, how long they could wait before giving oxygen, and how to titrate. This clinical knowledge was respected by physicians:

I think they often have an awareness of them (i.e., premature infants). They are bedside, and you notice quickly how an experienced nurse might say "hands off, he is a little high (in saturation), but he falls very low when he falls, or he gets fluctuations"-especially in phases when the BPD is under development and the infants have shunts - they (nurses) allow a greater fluctuation, so then you know what they are doing. (3-2)

The following excerpt shows how this long-term view of the infant influenced decision-making:

You have those infants; you turn up and down all the time to find just the window where the infant should be. And when you have probably used half the day doing this, and just at that moment when the saturation settles at $93-94 \%$ the physician comes, and then you get a sort of finger-pointing because you gave too much oxygen, but I have used all the day to find exactly this - it was not so easy to find exactly this balance. (2-6)

This illustrates how the physician made a quick decision based on a snapshot impression, while the nurse adjusted the oxygen for half of the day without an opportunity to articulate the observations and actions before the physician's oxygen reduction. This experience was confirmed by another nurse: "It was the same thing I experienced then; the doctor turned down the oxygen and just left. Then everything fell. Actually, I have experienced that several times" (2-4). The discussions revealed that a norm for oxygen treatment in the unit was to give as little oxygen as possible. A "good" intensive care chart should ideally illustrate a fraction of inspired oxygen $\left(\mathrm{FiO}_{2}\right)$ at $21 \%$. The nurses reported that they felt it was wrong to turn up the $\mathrm{FiO}_{2}$ and that a good nurse could produce a chart that reflected a reduction in $\mathrm{FiO}_{2}$.

\section{Discussion}

The physician-directed ventilator treatment in this NICU represents a great challenge in clinical reasoning because professionals must balance evidence and generally accepted healthcare practices with person-centered practice [33]. In relation to Hoc's [4] perspective on collaboration, interprofessional collaboration can be improved at all three levels. To achieve an improvement in quality of care, it is not sufficient only to determine what to improve; the caregivers also need to know how 
12

Collaboration on Ventilation of Newborns

Solberg, Hansen, \& Bjørk

Journal of Research in Interprofessional Practice and Education

Vol. 4.1

June 2014 to do it [34]. The discussion is therefore focused on achievable strategies that can improve interprofessional collaboration in NICUs.

\section{Collaboration at the planning level}

This study found that collaboration failed at the planning level because there were few arenas where the nurses and physicians could communicate and formulate plans together. Most nurses were not aware of the physicians' reasons for treatment. Good interprofessional collaboration defined in this study, and in other literature $[23,22]$, aims to share information, goals, and knowledge that will improve the choice of plans. In our study, NICU nurses communicated their assessments mostly during physician rounds but rarely in the pre-round conference. This lack of collaborative planning coincides with findings in a study that concluded that physicians and nurses could more frequently share their professional knowledge using a mutual approach to ensure continuity of care [33]. Broadened participation in patient rounds has been found to contribute to high-performance healthcare because it allows physicians and nurses to meet face to face and share information when collaborating in the decision-making process [23]. The pre-round conference could provide an opportunity to collaborate face-to-face regarding newborn ventilation problems. Following this argument, broader participation of the nurses in pre-round conferences could improve continuity of the ventilator treatment, which we found to be mostly managed with exercise of individual discretion and practice of care delivery [36]. Broader participation could secure information exchange and accommodate the physicians' need for nurse observations. The importance of using nurse observations in the decision-making process is supported by a Norwegian study in which the nurses' assessments were found to be more important for physicians than the physicians' assessments were for nurses [37]. Similar to nurses in this study, half of the nurses from 12 NICUs in the U.S. felt that their arguments were not well received, and they desired to be more involved in decision-making [13]. We do not know whether broader participation in the pre-round conference would be the best arena for nurses to share information with the physicians. The current preround conferences, in which one nurse is responsible for all the information exchange, prevent mutual exchange of information. Based on our findings, we suggest that the quality of care in the NICU may be improved using Gittels's [23] recommendation of giving professionals the opportunity to co-ordinate their tasks in meetings and to offer time to collaborate and express their assessments in the decision-making process.

\section{Collaboration at the action level}

In this study, communication was valued as the most important element in collaboration. Both physicians and nurses characterized the communication as varied, but the impression of good communication practice was highest among the physicians. The physicians' sense of predominantly good communication and decision-making processes was consistent with previous studies $[13,38,11]$. An evaluation using Hoc's [4] descriptions at the action level showed that communication in the studied 
Collaboration on Ventilation of Newborns

Solberg, Hansen, \& Bjørk

Journal of Research in Interprofessional Practice and Education

Vol. 4.1

June 2014
NICU has room for improvement. The hospital hierarchy negatively affects the communication between nurses and physicians [25], which may be why some of the nurses felt uncomfortable in voicing their opinions. This has been reported as a problem in other NICUs [13]. The professions of nursing and medicine represent different cultures in collaboration with respect to values, beliefs, attitudes, customs, and behaviours [39]. As a nurse or physician, it is important to know how to act when the professionals disagree [23]. To reach a mutual decision-making process, staff must develop skills that facilitate two-way communication [33]. Gittel [23] highlights teamwork skills because the co-ordination of patient care requires teamwork among the professions. Although caregivers may have experience and evidence knowledge, it may be difficult to achieve a commitment that enables co-operation in a team.

Communication regarding the ventilator setting was influenced by questions and information exchange in a unidirectional manner with little reciprocity. Highquality communication needs to be frequent, timely, accurate, and problem solving [23]. Weinberg et al. [3] reported that only two of 20 physicians reported frequent communication with nurses and that high levels of communication in patient care rarely occurred. Timely communication from a physician's view involved the nurses communicating signs and symptoms that the physician needed to know when the physician needed to know them [3]. Nurses often find problems in the ventilator treatment before the physicians; therefore, it is important that the nurses have courage to speak up.

The nurses also agreed that physicians relied on experienced nurses who could express factual knowledge about an infant's condition. This matches the findings in Weinberg's [3] study in which physicians trusted nurses when they communicated facts rather than opinions. Many of the nurses wanted to articulate small changes and discrete cues that they deemed necessary in the decision-making process. Recently, a review underscored the importance of establishing nonhierarchical and collaborative nurse-physician communication [40]. By using specific communication elements such as SBAR (situation, background, assessment, and recommendation) or a STICC protocol (situation, task, intent, concern, and calibrate), nurses could be heard because of the increased content of the communication [38]. Research on the use of protocols found that nurses became more effective in their roles [38], the time to present critical data was reduced [43], and the quality of care improved because the communication became more concrete and structured $[38,42]$. Skills in formal, clear, and timely communication are necessary for caregivers in NICUs but are seldom highlighted in training programs [43]. Improvement in communication skills is necessary for nurses and physicians, and training could involve role-play [41] or the use of a STICC protocol [38].

Because nearly all the respondents were frustrated by the physician-selected mode in the NICU, according to Gittel's theory [23], the subjectivity of the ventilator treatment was systemic and collective in nature. In general, both physicians and nurses want to collaborate and manage their care and treatment to provide the best care for newborn infants [23]. Collaboration practices are found to depend on the 
14

Collaboration on Ventilation of Newborns

Solberg, Hansen, \& Bjørk
Journal of Research in Interprofessional Practice and Education

Vol. 4.1

June 2014 caregiver's attitude, autonomy, independence, and knowledge [22,23]. Although caregivers wanted to promote their own convictions regarding care and treatment, they attempted to balance evidence-based knowledge and individual practice of care delivery [36]. To achieve improvements in the quality of care, performancemeasurement systems may assign accountability to the nurses' or physicians' roles [23]. A quality improvement program could set up cross-functional teams to recommend process improvements, which are found to prevent errors, improve co-ordination, and encourage the professions to move from finger pointing to problem solving [23].

\section{Collaboration at the meta level}

Physicians and nurses shared a common understanding that the rules of responsibility for ventilator treatment were restrictive and provided limited flexibility in practical treatment. Limited flexibility in the management of the ventilator treatment may hinder effective practice in reaching goals. More flexibility between the professions is found to improve practice because physicians and nurses can cover for each other and reduce the length of stay [23]. To enhance co-operation on the meta level, Hoc [4] recommends the creation of tools or guidelines. Specific guidelines in oxygen titration were found to be useful to caregivers in several NICUs in the U.S. [17]. A protocol to guide oxygenation and ventilator treatment may allow the allocation of responsibility to become more flexible and improve the utilization of the nurses' medical knowledge. Collaboration at the meta level might reduce the need for interruptions during physician rounds when an infant needs adjustment of the ventilator setting. One physician also expressed a concern about the use of guidelines versus the use of experience and feelings in the decision-making process. This is an issue addressed in a study from general intensive care [44]. Although the use of guidelines and protocols in patient work was found to support nurses in their decision-making, these tools did not replace the use of professional and ethical judgment.

There are several limitations to this study. More nurses than physicians participated to represent the employee ratio, which gave richer data from nurses than physicians. Participation was voluntary, and we do not know if the respondents differed in their views from non-respondents in any specific ways. Observation of the collaboration in the unit might have revealed other outcomes or might have supported our results. Because the first author is an intensive care nurse, the question also arises whether these results are valid when presenting data from the physician interviews. To ensure accuracy of the categories and themes from the analysis, we sought agreement among the co-researchers, who had both medical and non-intensive nurse backgrounds. As Barbour [45] suggested, the moderator attempted to utilize the interaction by presenting contrasting perspectives in the discussion.

Findings from this study indicate that nurses and physicians may improve the quality of care by advancing their communication strategies. Improved communication includes the formulation of plans together, the development of skills that facilitate two-way communication, and the use of guidelines to co-ordinate ventila- 
15

Collaboration on Ventilation of

Solberg, Hansen, \& Bjørk Newborns

tor treatment. Difficulties collaborating and challenges in co-ordinating intensive care are relevant to physicians and nurses in other countries as they manage problems related to treatment of critically ill patients whose lives are at stake. To improve oxygen and ventilator treatment for premature and sick newborn infants, further studies should develop and test interventions based on professionals' perceptions of relevant co-ordination strategies.

\section{Acknowledgements}

The authors are grateful to the Norwegian Premature Association and the Norwegian Extra Foundation for Health and Rehabilitation for their support of this study.

\section{Abbreviations \\ NICU $=$ Neonatal intensive care unit \\ SBAR = situation, background, assessment, recommendation \\ STICC = situation, task, intent, concern and calibrate \\ $\mathrm{FiO}_{2}=$ fraction of inspired oxygen \\ SIPPV = synchronized intermitted positive pressure ventilation}

\section{References}

1. Solberg, M.T., Bjork, I.T., \& Hansen, T.W. (2013). Adherence to oxygenation and ventilation targets in mechanically ventilated premature and sick newborns: A retrospective study. BMC Pediatrics, 13(1), 126.

2. Thomas, E.J., Sherwood, G.D., Mulhollem, J.L., Sexton, J.B., \& Helmreich, R.L. (2004). Working together in the neonatal intensive care unit: Provider perspectives. Journal of Perinatology, 24(9), $552-559$.

3. Weinberg, D.B., Miner, D.C., \& Rivlin, L. (2009). 'It depends': Medical residents' perspectives on working with nurses. The American Journal of Nursing, 109(7), 34-43.

4. Hoc, J.H. (2001). Towards a cognitive approach to human-machine cooperation in dynamic situations. International Journal of Human-Computer Studies, 54(4), 509-540.

5. Leonard, M., Graham, S., \& Bonacum, D. (2004). The human factor: The critical importance of effective teamwork and communication in providing safe care. Quality \& Safety in Health Care, 13(S1), i85-90.

6. Manojlovich, M., \& DeCicco, B. (2007). Healthy work environments, nurse-physician communication, and patients' outcomes. American Journal of Critical Care, 16(6), 536-543.

7. Schmalenberg, C., Kramer, M., King, C.R., Krugman, M., Lund, C., Poduska, D., \& Rapp, D. (2005). Excellence through evidence: Securing collegial/collaborative nurse-physician relationships, part 1. Journal of Nursing Administration, 35(10), 450-458.

8. Gittell, J.H., Weinberg, D., Pfefferle, S., \& Bishop, C. (2008). Impact of relational coordination on job satisfaction and quality outcomes: A study of nursing homes. Human Resource Management Journal, 18(2), 154-170.

9. Curley, G., Laffey, J.G., \& Kavanagh, B.P. (2010). Bench-to-bedside review: Carbon dioxide. Critical Care (London, England), 14(2), 220.

10. Saugstad, O.D., Sejersted, Y., Solberg, R., Wollen, E.J., \& Bjørås, M. (2012). Oxygenation of the newborn: A molecular approach. Neonatology, 101(4), 315-325.

Journal of Research in Interprofessional

Practice and

Education

11. Dunn, S., Cragg, B., Graham, I.D., Medves, J., \& Gaboury, I. (2013). Interprofessional shared decision making in the NICU: A survey of an interprofessional healthcare team. Journal of Research in Interprofessional Practice and Education, 3(1), 62-77.

12. Boos, V.D., Okah, F.A., Swinton, C.H., Wolff, D.M., \& Haney, B. (2010). The comprehensive care rounds: Facilitating multidisciplinary communication among caregivers of complex patients in the neonatal intensive care unit. Advances in Neonatal Care, 10(6), 301-306. 
16

Collaboration on Ventilation of Newborns

Solberg, Hansen, \& Bjørk

Journal of Research in Interprofessional Practice and Education

Vol. 4.1

June 2014
13. Profit, J., Etchegaray, J., Petersen, L.A., Sexton, J.B., Hysong, S.J., Mei, Minghua, \& Thomas, E.J. (2012). Neonatal intensive care unit safety culture varies widely. Archives of Disease in ChildhoodFetal and Neonatal Edition, 97(2), F120-F126.

14. Barbosa, V.M. (2013). Teamwork in the neonatal intensive care unit. Physical \& Occupational Therapy in Pediatrics, 33(1), 5-26.

15. Muhlethaler, V., \& Malcolm, G. (2012). Mechanical ventilation in the newborn: A simplified approach. Part 1: Intermittent positive pressure ventilation. Journal of Paediatrics and Child Health, 48(8), 649-652.

16. Johnson, K., Scott, S.D., \& Fraser, K.D. (2011). Oxygen use for preterm infants: Factors that may influence clinical decisions surrounding oxygen titration. Advances in Neonatal Care, 11(1), 8-14; quiz 15-16.

17. Bancalari, E., \& Claure, N. (2012). Control of oxygenation during mechanical ventilation in the premature infant. Clinics in Perinatology, 39(3), 563.

18. Theofilou, P., (2012). Communication among healthcare professionals: A central factor in patient outcome. Journal of Nursing \& Care, 1(1), 101.

19. Sugrue, C., \& Solbrekke, T.D. (2011). Professional responsibility: New horizons of praxis. London: Routledge.

20. Bosque, E. (2011). A model of collaboration and efficiency between neonatal nurse practitioner and neonatologist: Application of collaboration theory. Advances in Neonatal Care, 11(2), 108.

21. Hsieh, E., Ju, H., \& Kong, H. (2010). Dimensions of trust: The tensions and challenges in providerinterpreter trust. Qualitative Health Research, 20(2), 170-181.

22. D’Amour, D., Ferrada-Videla, M., San Martin Rodriguez, L., \& Beaulieu, M.-D. (2005). The conceptual basis for interprofessional collaboration: Core concepts and theoretical frameworks. Journal of Interprofessional Care, 19(S1), 116-131.

23. Gittell, J.H. (2009). High performance healthcare: Using the power of relationships to achieve quality, efficiency and resilience. New York: McGraw-Hill.

24. Havens, D.S., Vasey, J., Gittell, J.H., \& Lin, W.T. (2010). Relational coordination among nurses and other providers: Impact on the quality of patient care. Journal of Nursing Management, 18(8), 926-937.

25. Manojlovich, M. (2010). Nurse/physician communication through a sensemaking lens: Shifting the paradigm to improve patient safety. Medical care, 48(11), 941.

26. Pullon, S. (2008). Competence, respect and trust: Key features of successful interprofessional nurse-doctor relationships. Journal of Interprofessional Care, 22(2), 133-147.

27. Polit, D.F., \& Beck, C.T. (2012). Introduction to nursing research in an evidence-based practice enviroment. In Nursing research: Generating and assessing evidence for nursing practice. (pp. 16-23). Philadelphia, PA: Wolters Kluwer Health.

28. Krueger, R.A., \& Casey, M.A. (2009). Focus groups: A practical guide for applied research. Los Angeles, CA: Sage.

29. Patton, M.Q. (2002). Designing qualitative studies. In Qualitative research \& evaluation methods. (pp. 230-246). Thousand Oaks, CA: Sage Publications.

30. Kvale, S., \& Brinkmann, S. (2009). Interview analyses focusing on meaning. In Interviews: Learning the craft of qualitative research interviewing. (pp. 201-218). Los Angeles, CA: Sage.

31. Smithson, J. (2000). Using and analysing focus groups: Limitations and possibilities. International Journal of Social Research Methodology, 3(2), 103-119.

32. Sandelowski, M. (2012). The weakness of the strong/weak comparison of modes of inquiry. Research in Nursing \& Health, 35(4), 325-327.

33. Higgs, J. (2008). Clinical decision making and multiple problem spaces. In Higgs, J., Jones, M., Loftus, S., \& Christensen, N. (Eds.), Clinical reasoning in the health professions. (pp. 3-17). Amsterdam: Elsevier BH.

34. Auerbach, A.D., Landefeld, C.S., \& Shojania, K.G. (2007). The tension between needing to improve care and knowing how to do it. New England Journal of Medicine, 357(6), 608.

35. Manojlovich, M., \& Talsma, A. (2007). Identifying nursing processes to reduce failure to rescue. Journal of Nursing Administration, 37(11), 504-509.

36. Golec, L. (2009). The art of inconsistency: Evidence-based practice my way. Journal of Perinatology, 29(9), 600-602.

37. Krogstad, U., Hofoss, D., \& Hjortdahl, P. (2004). Doctor and nurse perception of inter-professional co-operation in hospitals. International Journal for Quality in Health Care, 16(6), 491-497.

38. Manojlovich, M., Antonakos, C.L., \& Ronis, D.L. (2009). Intensive care units, communication between nurses and physicians, and patients' outcomes. American Journal of Critical Care, 18(1), 21-30. 
17

Collaboration on Ventilation of

Newborns

Solberg, Hansen, \& Bjørk
39. Hall, P. (2005). Interprofessional teamwork: Professional cultures as barriers. Journal of Interprofessional Care, 19 (S1), 188-196.

40. Crawford, C.L., Omery, A., \& Seago, J.A. (2012). The challenges of nurse-physician communication: A review of the evidence. Journal of Nursing Administration, 42(12), 548-550.

41. Cunningham, N.J., Weiland, T.J., van Dijk, J., Paddle, P., Shilkofski, N., \& Cunningham, N.J. (2012). Telephone referrals by junior doctors: A randomised controlled trial assessing the impact of SBAR in a simulated setting. Postgraduate Medical Journal, 88(1045), 619-626.

42. Thompson, J.E., Collett, L.W., Langbart, M.J., Purcell, N.J., Boyd, S.M., Yuminaga, Y., Ossolinski, G., Susanto, C., \& McCormack, A. (2011). Using the ISBAR handover tool in junior medical officer handover: A study in an Australian tertiary hospital. Postgraduate Medical Journal, 87(1027), 340-344.

43. Boss, R., Urban, A., Barnett, M., \& Arnold, R. (2013). Neonatal critical care communication (NC3): Training NICU physicians and nurse practitioners. Journal of Perinatology, 33, 642-646.

44. Wøien, H., \& Bjørk, I.T. (2013). Intensive care pain treatment and sedation: Nurses' experiences of the conflict between clinical judgement and standardised care: An explorative study. Intensive and Critical Care Nursing, 29(3), 128-136.

45. Barbour, R.S. (2007). Doing focus groups. Los Angeles, CA: Sage. 